University of Nebraska - Lincoln

DigitalCommons@University of Nebraska - Lincoln

USDA National Wildlife Research Center - Staff Publications
U.S. Department of Agriculture: Animal and Plant Health Inspection Service

2012

\title{
Towards optimized population control efficiency in space and time: A modelling framework adapted to a colonial waterbird
}

\author{
Alban Guillaumet \\ Mississippi State University, albanguillaumet@trentu.ca \\ Brian S. Dorr \\ USDA/APHIS/WS National Wildlife Research Center, brian.s.dorr@aphis.usda.gov \\ Guiming Wang \\ Mississippi State University, gwang@cfr.msstate.edu
}

Follow this and additional works at: https://digitalcommons.unl.edu/icwdm_usdanwrc

Guillaumet, Alban; Dorr, Brian S.; and Wang, Guiming, "Towards optimized population control efficiency in space and time: A modelling framework adapted to a colonial waterbird" (2012). USDA National Wildlife Research Center - Staff Publications. 1142.

https://digitalcommons.unl.edu/icwdm_usdanwrc/1142

This Article is brought to you for free and open access by the U.S. Department of Agriculture: Animal and Plant Health Inspection Service at DigitalCommons@University of Nebraska - Lincoln. It has been accepted for inclusion in USDA National Wildlife Research Center - Staff Publications by an authorized administrator of DigitalCommons@University of Nebraska - Lincoln. 


\title{
Towards optimized population control efficiency in space and time: A modelling framework adapted to a colonial waterbird
}

\author{
Alban Guillaumet ${ }^{\mathrm{a}, \mathrm{b}, *}$, Brian Dorr ${ }^{\mathrm{c}}$, Guiming Wang ${ }^{\mathrm{a}}$ \\ a Department of Wildlife, Fisheries and Aquaculture, Mail Stop 9690, Mississippi State University, Mississippi State, MS 39762, USA \\ ${ }^{\mathrm{b}}$ Department of Biology, Trent University, Office D223, DNA Building, Peterborough, ON K9J 7B8, Canada \\ ' United States Department of Agriculture, Wildlife Services, National Wildlife Research Center, Box 6099, Mississippi State, MS 39762, USA
}

\section{A R T I C L E I N F O}

\section{Article history:}

Received 15 September 2011

Received in revised form 23 March 2012

Accepted 25 March 2012

\section{Keywords:}

Adaptive management

Human-wildlife conflict

Management efficiency

Metapopulation

Model

Simulation

\begin{abstract}
A B S T R A C T
The double-crested cormorant is a native North American waterbird that recently underwent a dramatic population expansion. Population control efforts in the USA and Canada attempt to mitigate cormorant damages to natural resources and aquaculture. However, there is currently no coordination among the various stakeholders involved in management activities as well as no attempt to optimize population control efficiency. In this paper, we present for the first time a spatially explicit stage-structured metapopulation model parameterized for the cormorant. We developed simulation tools to get insights into the efficiency gain that can be expected from a better planning of management activities in both space and time. A case study is presented, in which we randomized where (on which colonies) and when (which years) a pre-determined amount of management activities would occur on 4 or 8 of 16 active colonies arranged on a $4 \times 4$ or $2 \times 8$ spatial grid over a period of 8 years, including 2 or 4 management years. We calculated two indices measuring the location of management activities, namely the average date of management years and the average degree of peripherality of colonies undertaking management, together with two indices measuring the resulting correlation of management activities, i.e., the degree of clustering of management activities both in space and time. Different spatio-temporal configurations of management activities generally yielded different metapopulation trajectories. Room for improving management efficiency increased with the intensity of management activities. However, the greatest efficiency gains are to be expected when colonies are far from carrying capacity, while the majority of management operations are undertaken when colonies are near or at carrying capacity. Locations of management activities in space and time appeared more important than resulting spatio-temporal correlations to explain the dispersion of metapopulation trajectories. When colonies were far from their individual carrying capacity, management was more efficient when applied earlier (a consequence of delayed reproductive maturity) and to more central colonies (due to greater immigration). The situation was more complex when colonies were closer to or at carrying capacity. Our modelling framework is flexible enough to allow more complex scenarios to be investigated in the future.
\end{abstract}

(C) 2012 Elsevier B.V. All rights reserved.

\section{Introduction}

The double-crested cormorant (Phalacrocorax auritus, hereafter cormorant) is a large native North American piscivorous waterbird. The dramatic increase in cormorant abundance in recent decades (Wires and Cuthbert, 2006) has led to increased human-cormorant conflicts due to documented and alleged impacts on recreational fishing, aquaculture, vegetation, and competition with other colonial waterbirds (Taylor and Dorr, 2003).

\footnotetext{
* Corresponding author at: Department of Biology, Trent University, Office D223, DNA Building, Peterborough, ON K9J 7B8, Canada.

E-mail address: albanguillaumet@trentu.ca (A. Guillaumet).
}

Population control efforts were progressively implemented in the United States of America (USA) and Canada to mitigate these conflicts. Cormorant management techniques include harassment at roosting and breeding sites, culling of breeding and wintering cormorants, egg oiling to prevent hatching and re-laying, and nest destruction or removal. In Canada, cormorants are not federally protected, so management policy varies by province. In Ontario for instance, authorization to destroy nests of cormorants is issued on a case-by-case basis by the Ontario Ministry of Natural Resources (2006), but landowners may kill cormorants to protect their own property from cormorant damage without a permit. In the USA in 1998, the Aquaculture Depredation Order (AQDO; 50 CFR 21.47) allowed the lethal removal of cormorants at aquaculture facilities without a federal permit in 13 states. Since 2003, cormorants also have been managed under the Public Resource Depredation Order 

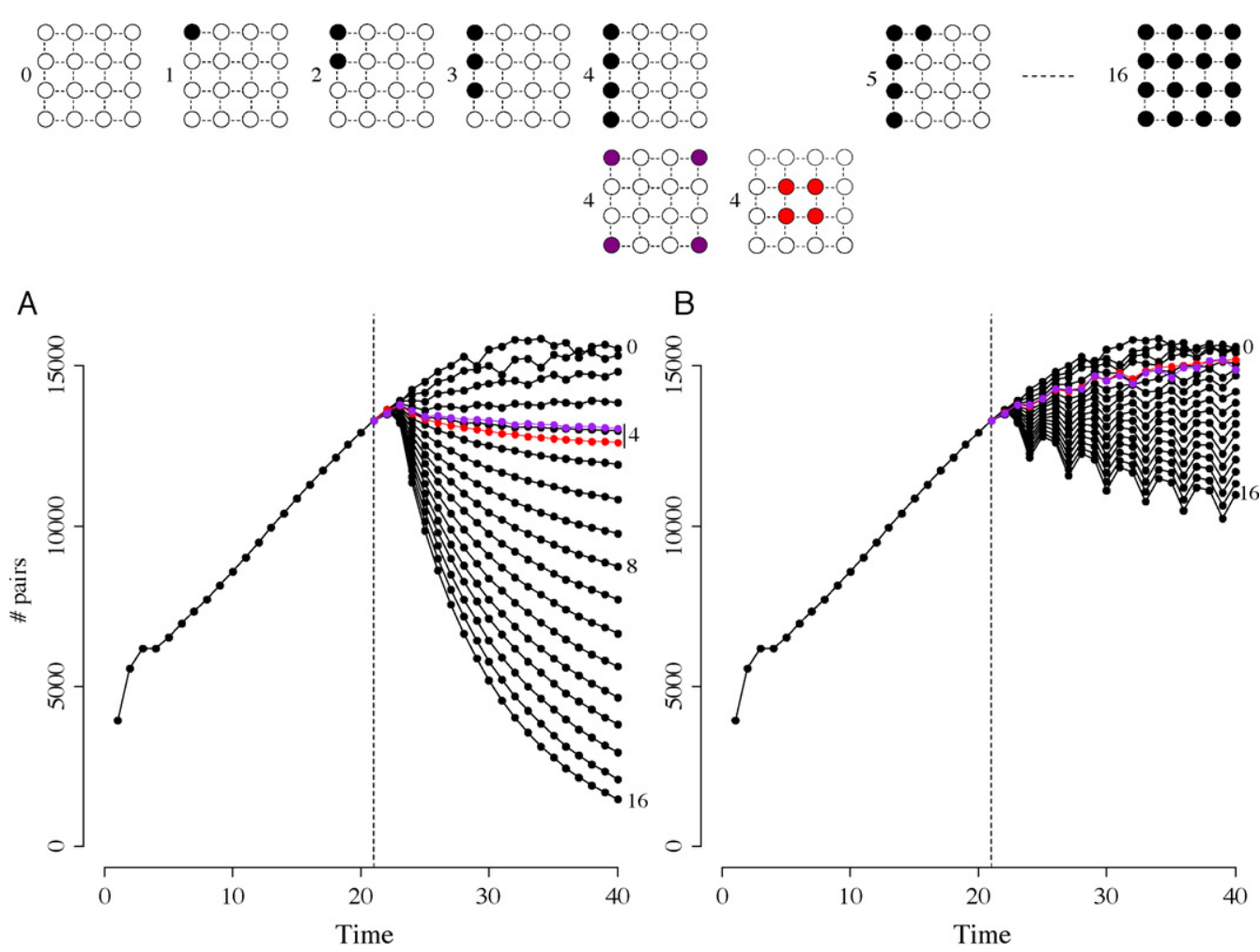

Fig. 1. Schematic representation of the model and example of model application showing the dynamics of 16 colonies ( 4 rows $\times 4$ columns). Management is $80 \%$ of eggs oiled with no culling, starting at $T m=21$, of a 40 year period. Part A has egg oiling occurring every year and part B egg oiling occurs every three years. All colonies are active and have a carrying capacity of $K p=1000$. Each black curve represents the total number of breeding pairs in the metapopulation, with the number of managed colonies varying between 0 and 16 (the position of managed colonies is represented by a black dot in upper part, first row). We also show the dynamics of three ' 4 managed colonies' scenarios with alternative spatial designs: purple (position_mg $=1.44, S C=0.29$ ), black (position_mg $=1.32, S C=0.60$ ), and red (position_mg $=1.00, S C=0.88$ ). See text for details, including variable descriptions. Other parameter values were taken from the "main" analysis in Appendix A.

(PRDO; 50 CFR 21.48), which authorizes the lethal take of cormorants and egg-oiling or nest destruction by federal, state, and tribal officials without a permit in 24 states.

Management efforts, and the associated costs, are currently substantial in North America. In the USA during 2009, a total of 47,190 cormorants were killed and 32,296 nests oiled or destroyed (Terry J. Doyle, United States Fish and Wildlife Service, unpublished data). The total cost of the United States Department of Agriculture, Wildlife Services, cormorant control program in central New York State alone from 1998 to 2005 was approximately \$3.5 million (Shwiff et al., 2009).

Literature on insect pest management suggests that coordinated decisions among crop growers may improve returns (Byers and Castle, 2005; McKee, 2011). Optimization of management in space and time may be important to mitigate the impact of a pest (Zhang et al., 2010; McKee, 2011; Parsa et al., 2011). For mobile pests, management at large spatial scales may be more effective and preferable to the uncoordinated field-by-field approach (Vreysen et al., 2007). Despite the knowledge gained from other pest management experiences and theoretical models, there currently is no population-level management strategy for the cormorant.

Due to the ongoing and intensive nature of cormorant management in North America we explored a modelling framework that can be used to inform cormorant management strategies. Specifically, our aims were as follows: (i) to present a deterministic stage-structured metapopulation model parameterized for the cormorant. Our model represents a step beyond the works of Blackwell et al. (2002) who developed a single population model and Duerr (2007) whose metapopulation model, parameterized for cormorant colonies in the Lake Champlain, lacked an explicit spatial context which is necessary to accommodate distance-dependent dispersal as evidenced in the closely related great cormorant (Henaux et al., 2007); (ii) as our model was designed to be an effective strategic and management decision tool, we also developed simulation tools to gain insight into the efficiency gain that could be expected from a better coordination of management activities in space and time; (iii) our modelling framework was applied to a case study in which we investigated the relative importance of the spatiotemporal location and degree of clustering of specific management activities on management efficiency.

\section{Materials and methods}

\subsection{The model}

\subsubsection{Spatial structure}

The spatial structure consists of a grid of $i=1$ to $n r$ rows and $j=1$ to $n c$ columns (all parameter definitions and values are given in Appendix A). Breeding colonies, when present, are located at grid intersections, and the number of breeding colonies ncol ranges from 2 to $n r \times n c$ (Fig. 1). We assume the diameter of colonies to be negligible in comparison with the distance between colonies. The shortest distance between two rows or two columns corresponds to one unit of distance. All suitable habitats (colonies) are occupied at the beginning of the simulation (i.e., founding of new colonies is not allowed), but local extinction-recolonization dynamics may occur as a result of management activities.

\subsubsection{Local demography}

Following Blackwell et al. (2002) and Duerr (2007) the breeding population consists of three age groups: year 1 subadults were juveniles produced during the previous breeding season, year 2 
subadults, and a composite age class consisting of adults $\geq 3$ years old. Below we describe the demography of colony $i, j$ at time $t(i, j$, $t$ indices are omitted in the equations).

Culling may occur before and/or after reproduction takes place, and the proportion of birds culled (cs) may be sex-specific. Because culling is suspected to impact primarily the males in both the breeding and nonbreeding grounds (Bedard, 1995; Strickland et al., 2011), our model assumes either an even or a female-biased sexratio. The hatching success is taken to be $h=1-\operatorname{egg} D$, where $\operatorname{egg} D$ is the proportion of eggs laid that do not hatch as a result of management activities. In this paper, we mainly consider egg oiling, as this is the most frequently used method (A. Guillaumet et al., unpublished data), and because it results in almost $100 \%$ of eggs that do not hatch (Shonk et al., 2004). Nest destruction, or predation due to disturbance, can easily be accommodated, but the proportion of eggs lost is more difficult to estimate, because a fraction of cormorants will re-nest and/or re-lay eggs (Duerr et al., 2007).

Density-dependent regulation is modelled using one of two methods. The first method is based on Frederiksen et al. (2001). The breeding proportion of year 2 subadults and adults (b2 and $b 3$, respectively) are density-independent $(b(0)$ values) when the number of potential breeding pairs (pot_pairs) is below a given threshold thr_DDb, pot_pairs being calculated as pot_pairs $=b 2(0) \times B 2 m+b 3(0) \times B 3 m$, where $B 2 m$ is the number of year 2 subadult males and $B 3 \mathrm{~m}$ the number of adult males present. When pot_pairs $\geq$ thr_ $D D b$, breeding proportions take the form $b=b(0) \cdot e^{-D D b(\text { pot_pairs }-t h r D D b)}$, with $D D b$ being the slope of the density-dependent relationship. Fertility coefficients ( $f 2$ and $f 3$, see Appendix A) are density-independent $(f(0)$ values) when the actual number of breeding pairs (act_pairs) is below a given threshold thr_DDf, act_pairs being calculated as act_pairs $=\mathrm{b} 2 \times \mathrm{B} 2 \mathrm{~m}+\mathrm{b} 3 \times \mathrm{B} 3 \mathrm{~m}$. When act_pairs $\geq$ thr_DDf, fertility coefficients take the form $f=f(0) \cdot e^{-D D f(a c t \text { pairs }}-$ thr.DDf), with $D D f$ being the slope of the density-dependent relationship. The number of juveniles produced $(B 0)$, is calculated as $B 0=h \times(b 2 \times f 2 \times B 2 m+b 3 \times f 3 \times B 3 m) ; B 0 m=B 0 f=1 / 2 \times B 0$.

The first method does not guarantee that the number of breeding pairs will not exceed a certain value $(K p)$, which is the carrying capacity of the number of pairs (e.g., corresponding to the number of nest sites available). We thus implemented a second method, where the actual number of breeding pairs is calculated as act_pairs $=p$ _breed $\times$ pot_pairs, where $p$ _breed, the proportion of pairs actually breeding, is 1 if pot_pairs $\leq K p$, and (Kp/pot_pairs) otherwise. We also accommodated the possibility that the total number of individuals in the breeding colony after reproduction, summing over all age classes and sex (noted $B$ ), did not exceed a certain value $K b$, a carrying capacity corresponding to the quantity of resources available around the colony. The potential number of juveniles produced (pot_B0), was thus calculated as pot $B 0=p \_$breed $\times h \times(b 2(0) \times f 2(0) \times B 2 m+b 3(0) \times f 3(0) \times B 3 m)$; $B 0=$ pot $B 0($ if $B<K b)$, but $B 0=$ pot_ $B 0-d($ if $B \geq K b)$, where $d=B-K b$ is the number of deaths necessary to keep $B \leq K b$.

\subsubsection{Dispersal}

Following Lebreton et al. (2003), we assume a higher annual dispersal rate for pre-breeding dispersal (m_natal, for cormorants less than 3 years-old which is the usual age of first reproduction) than for cormorants aged $3+$ years-old ( $m_{-}$breed). Although estimates available in the literature may not be generally applicable, as dispersal is a complex trait conditional on the interaction of individual, social and environmental causes (Martin et al., 2008), we used dispersal estimates described by Duerr (2007) and by Henaux et al. (2007) for the great cormorant as our references.

To account for potentially higher dispersal rates due to densitydependence effects (Doligez et al., 2003) or management activities (Duerr et al., 2007; Pope et al., 2007), natural dispersal rates of all ages classes (except juveniles) can be augmented by the parameter psi $D D$ when $b<b(0)$ or $p$ breed $<1$, psi_egg $D$ when $\operatorname{egg} D>0$, and by psi_cs when $c s>0$. Dispersing individuals are allocated to another colony with a probability inversely related to the distance to the source colony (e.g., Hansson et al., 2002). If the distance between the focal colony $f$ and another colony $g$ is $d_{f, g}$, the proportion of dispersing cormorants from $f$ actually going to $g$ is calculated as: $e^{-\left(d_{f, g} / 2\right)} / \sum_{m \neq f} e^{-\left(d_{f, m} / 2\right)}$, where the denominator is the sum (over $n c o l-1$ ) of the distances between the focal colony $f$ and any other colony $m$ of the metapopulation. We assume that the selection of a new breeding colony (dispersal) occurs between the end of the reproduction period and fall migration, at a period when cormorants frequently visit other colonies (Guillaumet et al., 2011).

\subsubsection{Migration and overwinter survival}

Overwinter survival rate is calculated as $s \times(1-c w)$, where $s$ is the natural survival rate, and $c w$ is the probability of being culled in the non-breeding area. The model accommodates three migration strategies: (1) no migratory connectivity; $c w$ does not depend on the spatial position of the breeding colony; (2) parallel migration; $c w$ may vary according to the longitude, i.e., each column $j$ may have a different $c w_{j}$; (3) migratory divide; two different $c w_{j}$ are allowed, one for each side of the migratory divide. We assume that all individuals (including first years) come back to the breeding colonies, so that non-breeders contribute to deplete the resources, affecting the $B / K b$ ratio (see above).

\subsection{Simulations}

We simulated $4 \times 4=16$ active colonies over a period of 8 years. For each simulation, we started by picking at random the colonies that would undergo management; 4 or 8 colonies were selected in this way (variable $=n b_{\_}$col_mg). We calculated $S C$, an index of spatial correlation of management, inversely related to the average geographic distance between managed colonies (the more clustered the managed colonies, the higher the index). We also calculated position_mg, the average position of managed colonies on the grid, as follows: (i) we gave each colony an index of peripherality; and (ii) position_mg was calculated as the average peripherality of managed colonies. The index of peripherality was 1 for the four central colonies, 1.44 for the four most peripheral (corner) colonies, and 1.20 for the remaining eight colonies (Fig. 1). These values derived from the dispersal transition matrix $(D)$ for each colony: $\max (D)$ was used as a proxy for the peripherality, the rationale being that more central colonies will distribute their migrants more evenly among neighboring colonies, yielding a lower $\max (D)$ value as elements of $D$ sum up to 1 . The resulting indices were adjusted so that the value for central colonies was 1 .

Next, we picked at random 2 or 4 years of the 8 years simulated during which management would be applied on the randomly selected colonies (variable $=n b_{-} y r_{\_} m g$ ). We calculated $T C$, an index of temporal correlation of management, inversely related to the average number of years between management years (the more clustered the management years, the higher the index). We also calculated date_mg as the average of the management years, taking higher values when management occurred later during the study period. Finally, we assigned the severity of management activities (sev_mg) among two options: low (culling in summer $=0.1$, and egg oiling $=0.4$ ), or high (culling in summer $=0.2$, and egg oiling $=0.8$ ). We calculated the number of breeding pairs at the end of the simulation period as an estimate of the total metapopulation size $($ variable $=$ metapop_size $)$.

We thus investigated 8 different scenarios of management intensity ( $2 n b \_c o l \_m g$ values $\times 2 n b \_y r \_m g$ values $\times 2 s e v \_m g$ values). Each scenario was investigated by performing a series of 500 
Table 1

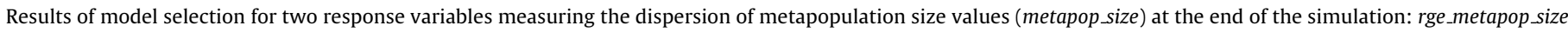
is the range of metapop_size values, and $C V$ the coefficient of variation of metapop_size.

\begin{tabular}{|c|c|c|c|c|}
\hline Response variable & Simulation factor & Explanation & t-value & $P$-value \\
\hline \multirow{6}{*}{ rge_metapop_size } & nb_col_mg & \# colonies managed & 3.02 & $<0.01$ \\
\hline & nb_yr_mg & \# years of management & 1.41 & 0.16 \\
\hline & sev_mg & Severity of management activities & 3.24 & $<0.01$ \\
\hline & DD_meth & Density-dependence method & 2.95 & $<0.01$ \\
\hline & disp & Dispersal magnitude & 1.70 & 0.09 \\
\hline & $\log ($ inits $)$ & log of initial population size & 14.72 & $<0.001$ \\
\hline \multirow{5}{*}{$\log (100 \times C V+1)$} & nb_col_mg & \# colonies managed & 5.83 & $<0.001$ \\
\hline & $n b_{-} y r_{-} m g$ & \# years of management & 1.85 & 0.07 \\
\hline & sev_mg & Severity of management activities & 5.55 & $<0.001$ \\
\hline & DD_meth & Density-dependence method & 3.21 & $<0.01$ \\
\hline & $\log$ (inits) & log of initial population size & -20.59 & $<0.001$ \\
\hline
\end{tabular}

simulations. To assess the sensitivity of the results to initial population size and to the choice of a specific density-dependent model and dispersal values, simulations for the 8 different management intensity scenarios were run for: (1) both density-dependent methods (DD_meth); (2) for two combinations of dispersal values (disp): low and high (see Appendix A for values); and (3) for initial population size (inits) drawn from a Poisson distribution with parameter $\lambda=10,50,100,200,500$ or 1000 . In practice, each sex and age category of each colony received an average of $\lambda$ individuals at $t=1$; the number of potential pairs at $t=1$ was inferior to the carrying capacity $K p$ in all but the last scenario $(\lambda=1000)$. Altogether, we thus conducted 8 (management intensity) $\times 6$ (initial population sizes) $\times 2$ (density-dependent methods) $\times 2$ (dispersal magnitude $)=192$ run of 500 simulations. Each run yielded a vector $(n=500)$ of each explanatory factor (SC, position_mg, TC, date_mg) and the resulting vector $(n=500)$ of the response variable (metapop_size).

We also performed two control analyses replicating these 192 run of 500 simulations, while modifying two other types of parameters: (1) 16 active colonies were arranged in 2 rows of 8 colonies (instead of $4 \times 4$ ); and (2) we used a different source of (higher) survival values (Appendix A).

\subsection{Statistical analyses}

For each run of 500 simulations, we first calculated the range of the response variable metapop_size (rge_metapop_size = difference between maximum and minimum metapop_size values) as a measure of the impact of the different management strategies (larger dispersion implies a larger potential to improve management efficiency). Using the standard deviation of metapop_size instead of rge_metapop_size yielded similar results (not shown). However, since standard deviation may scale proportional to the mean (e.g., Lande, 1977), we also calculated the coefficient of variation of metapop_size ( $C V=$ standard deviation/mean) as a normalized measure of dispersion.

Both rge_metapop_size and $C V$ were used as new (independent) response variables to identify, using linear models, which of the simulation factors were driving the dispersion of metapopsize values: number of colonies managed ( $n b_{c}$ col_mg), number of years of management (nb_yr_mg), severity of management activities (sev_mg), density-dependence method (DD_meth), dispersal magnitude (disp), and initial population size (inits). Both $C V$ and inits were log-transformed prior to analyses to increase linearity $(\log (100 \times C V+1)$ and $\log ($ inits $)$, respectively). We used a backward + forward stepwise selection procedure and AIC criterion to select the best model. Because the assumptions of linear models (normality of residuals and homoscedasticity) were rejected at the usual significance level (5\%), we also conducted non-parametric analyses to check the robustness of our conclusions (Wilcoxon rank-sum tests for binary variables, generalized additive models for $\log$ (inits)). As conclusions were identical, we only provide the results of linear models.

The role of explanatory factors was then evaluated as follows. First, for each simulation run we built a full (linear) model for metapop_size containing date_mg, position_mg, SC, TC and the interaction between SC and TC. We calculated the coefficient of determination $R_{\text {full }}{ }^{2}$ and Student's $t$-test values for each these five covariates. Second, we built a model containing only location variables date_mg and position_mg and calculated the coefficient of determination $R_{\mathrm{loc}}^{2}$; the statistic $R_{\text {full }}^{2}-R_{\mathrm{loc}}^{2}$ gave us the unique contribution due to the correlation of management activities in space and time. Third, we used Student's $t$-test values, estimated from the full model, as five new (independent) response variables to investigate, using linear models, which of the simulation factors were responsible for the negative or positive influence of each explanatory factor on metapop_size, using the same stepwise selection procedure as described above (Appendix B for further details). Modelling and statistical analyses were performed with R version 2.7.2 (@ The R Foundation for Statistical Computing).

\section{Results}

The range of the response variable metapop_size (variable $=$ rge_metapop_size) was 0-3268 (mean of rge_metapop_size $=712, \mathrm{SD}=629, n=192)$. Rge_metapop_size was strongly positively correlated with initial population sizes (Table 1 , Fig. 2), with the latter being strongly positively correlated with final metapopulation sizes (Pearson correlation coefficient on log-log scale, $r=0.99, P<0.001)$. In addition, the range of metapop_size increased with the intensity of management activities (Table 1: $n b_{-}$col_mg and sev_mg; although retained in the best model, $n b_{-} y r_{\_} m g$ did not reach significance), and was also influenced by the density-dependence method.

The coefficient of variation of metapop_size varied from 0 to $7.54 \%$ (mean of $C V=3.07, \mathrm{SD}=1.88, n=192$ ). Although $C V$ and rge_metapop_size were impacted by the same simulation factors (Table 1), CV (unlike rge_metapop_size) was negatively correlated with initial population sizes (Fig. 2C).

Our candidate explanatory factors explained a fraction of this dispersion. The coefficient of determination of the full model $\left(R_{\mathrm{full}}{ }^{2}\right)$ was $28.3 \%$ on average ( $S D=24.0$, range $=0.4-93.2, n=192$ ), while $R_{\mathrm{full}}{ }^{2}-R_{\mathrm{loc}}{ }^{2}$, the unique contribution due to the correlation of management activities in space and time (SC, TC and SC $\times$ TC) was only $4.0 \%$ on average $(\mathrm{SD}=6.9$, range $=0.0-41.9$ ).

The explanatory power of each factor is examined in Fig. 3, showing the histograms of Student's $t$-test values. Most $t$-values for $S C$ and $S C \times T C$ are in between -2 and +2 (non-significance thresholds), suggesting that these factors are generally not 

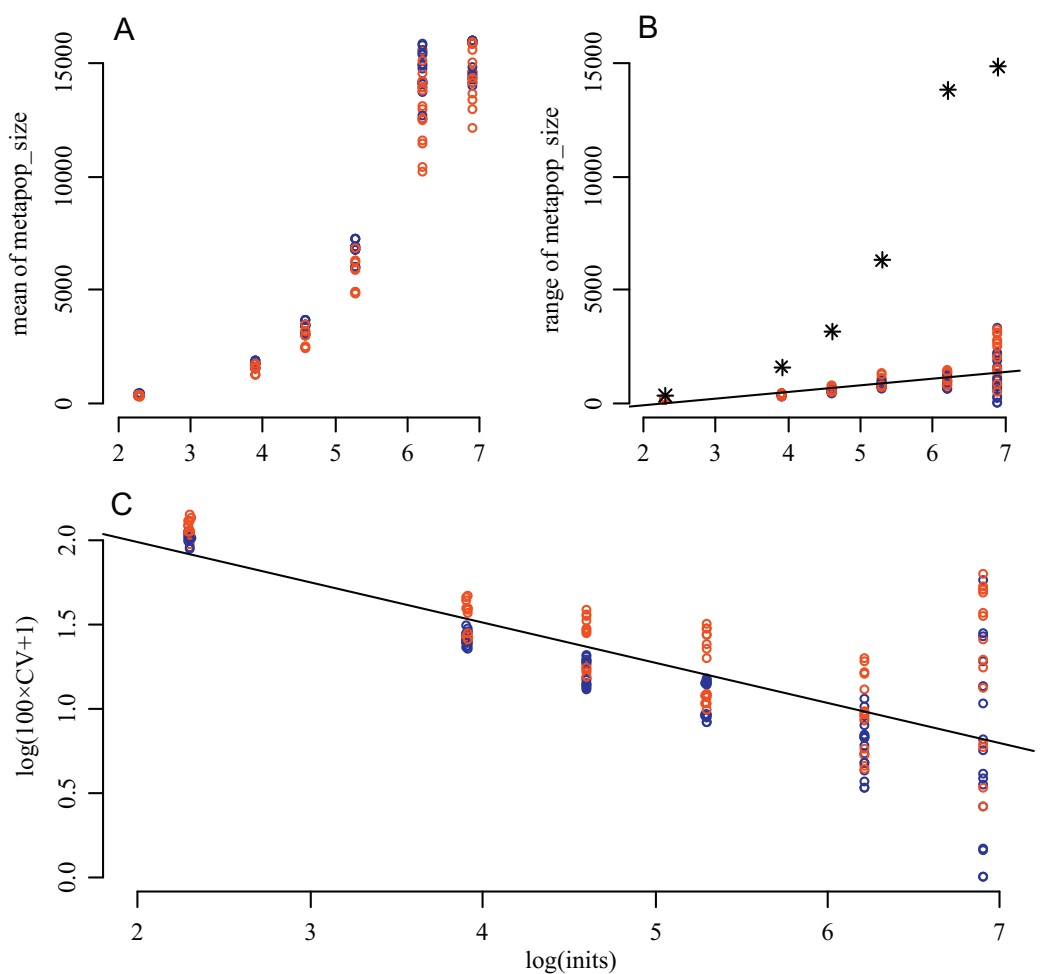

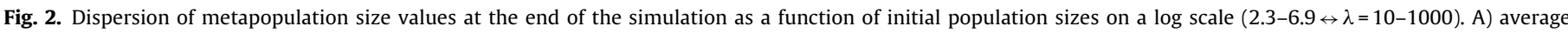

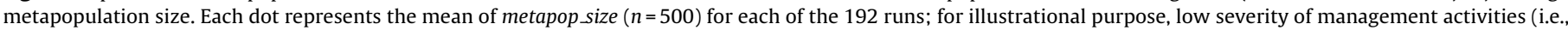

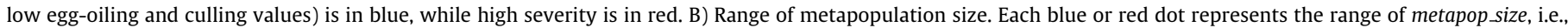

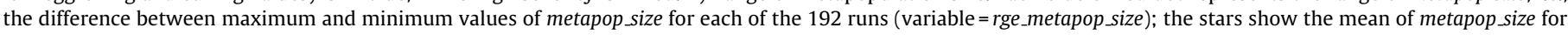

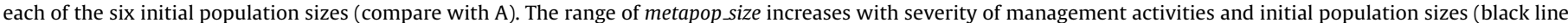

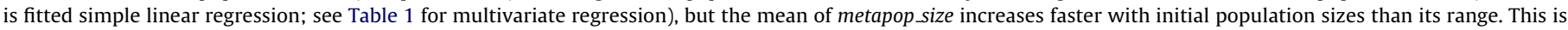
illustrated by the coefficient of variation of metapop_size $(\mathrm{CV})$ which decreases with initial population sizes $(\mathrm{C})$.

important. T-values for date_mg, position_mg and TC are slightly skewed towards negative values and positively correlated, the latter likely because they are strongly influenced by initial values. Larger initial values generally resulted in a more negative impact of these factors on the total metapopulation size (Fig. 3). The variable inits was by far the most significant simulation variable selected by the stepwise regression for date $m g\left(F_{1,188}=344.2, P<0.001\right)$ and position_mg $\left(F_{1,187}=160.7, P<0.001\right)$; hence the very strong correlation between $t$-values for date_mg and position_mg (Pearson's correlation coefficient $r=0.79$ ). For $T C$, inits was also the most significant variable $\left(F_{1,187}=94.4, P<0.001\right)$, but other simulation factors also played an important role, particularly the dispersal magnitude (disp: $F_{1,187}=55.3, P<0.001$ ) and the number of colonies managed ( $n b \_c o l \_m g: F_{1,187}=30.9, P<0.001$ ); hence the lower correlation between $t$-values for TC on one hand, and $t$-values for date_mg or position_mg on the other hand $(r=0.35-0.44)$. Stepwise regression results are detailed in Appendix B.

The two control analyses yielded similar results and so are not detailed. For instance, the coefficient of variation of the response variable metapop_size remained around $3 \%$ : $($ mean $=3.08, S D=1.88$ for the $2 \times 8$ spatial design, mean $=2.88, S D=2.12$ for the "higher survival" control). Both $R_{\text {full }}{ }^{2}$ and $R_{\text {full }}{ }^{2}-R_{\text {loc }}{ }^{2}$ were also very similar: for the $2 \times 8$ spatial design, $R_{\text {full }}{ }^{2}=29.7 \%$ on average $(\mathrm{SD}=25.0$ ), $R_{\text {full }}{ }^{2}-R_{\text {loc }}{ }^{2}=3.8 \%$ ( $\left.\mathrm{SD}=6.5\right)$; for the "higher survival" control, $R_{\text {full }}^{2}=29.1 \%(\mathrm{SD}=22.6), R_{\text {full }}{ }^{2}-R_{\text {loc }}{ }^{2}=4.3 \%(\mathrm{SD}=7.8)$. The main difference between treatments was observed when colonies were close to or at carrying capacity. In particular, while management was more efficient when applied later for the main analysis and $2 \times 8$ control, the reverse was generally observed for the "higher survival" control (Fig. 3, Appendices B and C).

\section{Discussion}

In this paper, we presented a metapopulation model parameterized for the double-crested cormorant, a species subjected to population control efforts in the USA and Canada. As an example of application, we designed a simulation study to provide insight into the efficiency gain that could be expected from a better planning and coordination of management operations. For every run of simulations (for a total of 192) we manipulated where (on which colonies) and when (which years) a pre-determined amount of management activities would occur, while all other parameters were kept constant. In turn, this choice determined how correlated the management activities were both in space and time.

We observed a non-null dispersion of metapopulation size values at the end of the simulation period for all but 2 runs, indicating that in the vast majority of situations different spatiotemporal configurations of management activities yielded different metapopulation trajectories. The most important source of dispersion was initial population sizes (Table 1). Although absolute dispersion increased with initial population sizes (Fig. 2B), the coefficient of variation followed an inverse trend (Fig. 2C). Arguably, the largest efficiency gains are thus to be expected when colonies are far from carrying capacity, while the majority of management operations are undertaken when colonies are near or at carrying capacity. We also found that both absolute and normalized dispersion significantly increased with the number of colonies managed and the severity of management operations, i.e., proportion of eggs oiled and cormorant culled (Table 1, Fig. 2). In other words, room for improving management efficiency increases with intensity of management activities. 

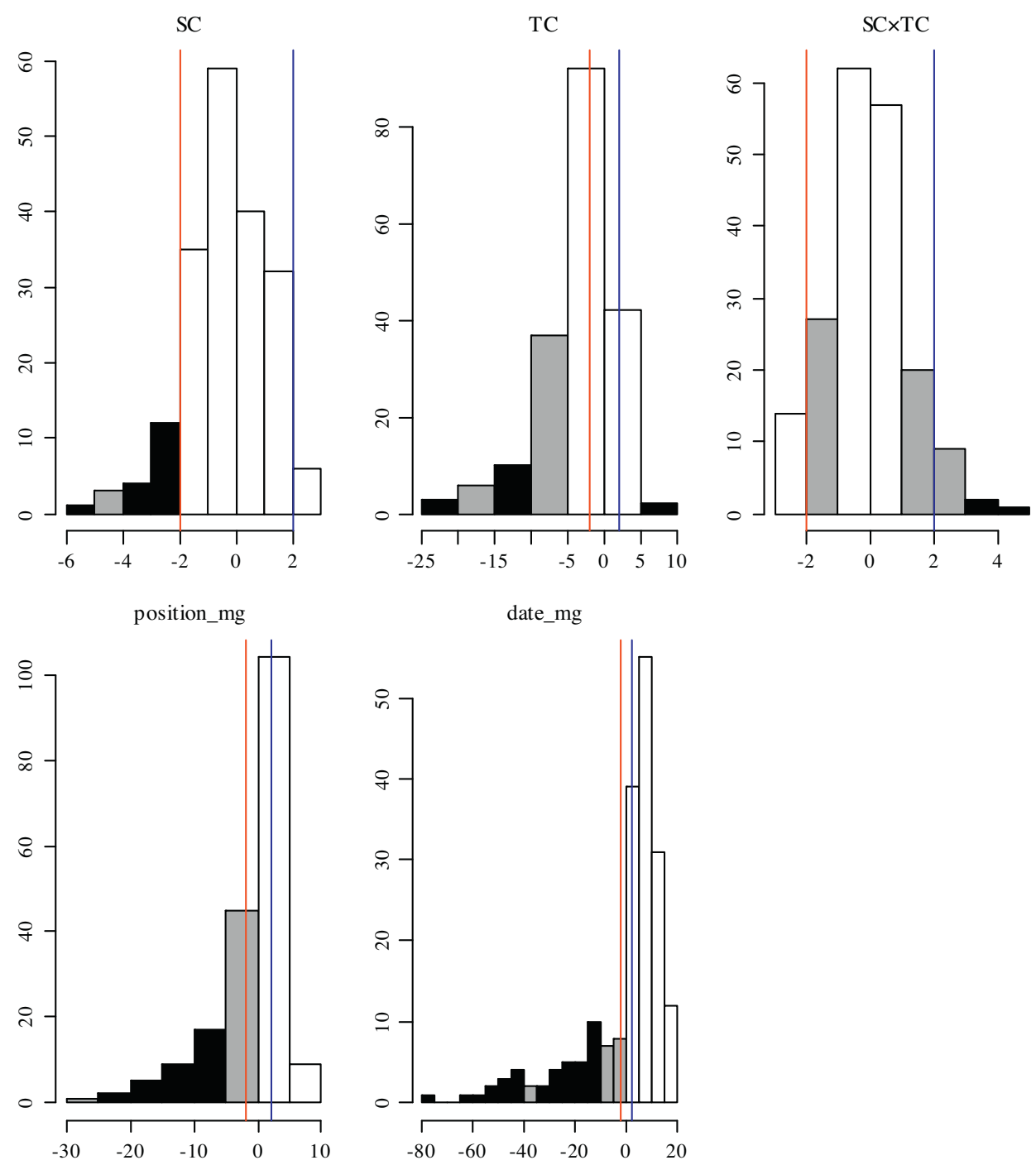

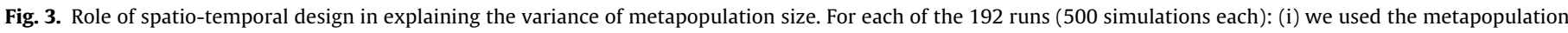

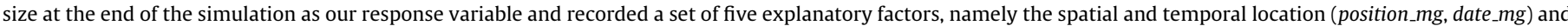

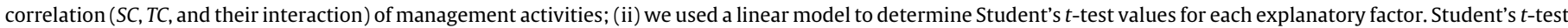

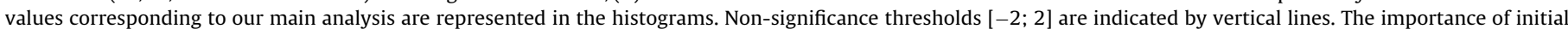

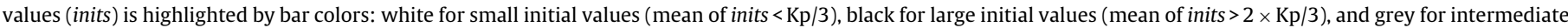
initial values; see text and Appendices A and B for details.

We further attempted to identify which spatio-temporal configurations were the most efficient. A comprehensive analysis of the mechanisms involved was beyond the scope of this study, so we restrict ourselves to some simple hypotheses. Coefficients of determination suggested that actual locations of management activities in space and time (position_mg and date_mg) were more important than resulting spatio-temporal correlations (SC, TC and $S C \times T C)$. When colonies were small and far from their individual carrying capacity, management was more efficient when applied earlier and to more central colonies (Fig. 3). Later management appears less efficient because it allows more time for the colonies to go through the steep phase of exponential population growth. Juveniles produced early become breeding adults, and the difference in age structure is not entirely counterbalanced by subsequent treatments. For the spatial dimension, peripheral colonies tend to receive a smaller fraction of the dispersing birds than more central colonies for purely geographical reasons. For instance, in the case where all colonies send out a same amount of dispersing birds, the four most peripheral colonies receive each $\sim 5.1 \%$, and the four central colonies each $7.5 \%$, of those dispersing birds $(4 \times 4$ design).
Hence, when regulation is essentially due to management activities (colonies far from carrying capacity), the population control is more efficient when management occurs on central colonies that grow faster owing to larger immigration.

The situation was more complex when colonies were close to or at carrying capacity, when population regulation is an interplay between natural density-dependence and anthropic disturbance. The fact that more, potentially counter-acting, factors were involved could account for the smaller relative impact of the spatio-temporal design (Fig. 2C). In our main design and the $2 \times 8$ control, the management was generally more efficient when applied later and to more peripheral colonies and, all else being equal, when management years and managed colonies tended to be clustered together (Fig. 3, Appendix C). Highly non-linear effects appear in the "higher survival" control, as illustrated by the date of management. Again, management was more efficient when applied earlier for small initial values (colonies far from carrying capacity), but in this scenario this was true also for large (but not intermediate) initial values (Appendix C). 
Of course, choices made in this paper to keep the model and the simulation design reasonably simple and tractable may have influenced our results to some extent. Caution should be exercised when trying to reach conclusions beyond the strict simulation conditions we used here. However, our modelling framework is flexible enough to allow additional sophistication of the model as well as more complex, real-life scenarios to be investigated in the future. For instance, future versions of the model could accommodate the possibility of density-dependent survival (Frederiksen and Bregnballe, 2000; but see also Frederiksen et al., 2001) and the possibility that cormorants, particularly first-time breeders, use public information to select a breeding colony (Henaux et al., 2007). More realistic scenarios may include irregular spatial grids with unequal spacing between active colonies, and the fact that different colonies may have different carrying capacities and/or be at different stages of their dynamics.

Because the number of such possible simulations is almost infinite, we believe that our simulation tools could find their most relevant application during the preparation of strategic management plans at local or regional scales, when managers and scientists can use real-life estimates of spatial parameters while simultaneously assessing the consequences of uncertainty in other model parameters. The model could be used to evaluate management intensity and structure to optimize desired outcomes, but management intensity could also be tuned relative to risk of undesirable management outcomes such as colony extirpation or metapopulation collapse. Because the model has been parameterized using a wide array of intra-, but also inter-specific data (great cormorant: see Appendix A), not necessarily relevant to actual local or regional context, our modelling tools would be especially valuable when incorporating feedback from monitoring programs within an adaptive management setting (e.g., Lyons et al., 2008).

\section{Acknowledgment}

We are grateful to three anonymous reviewers for their constructive comments on our manuscript. This project was funded by the USDA Wildlife Services, National Wildlife Research Center, and the Jack. H. Berryman Institute (Eastern Unit). G.M. Wang was partially supported by the Forest and Wildlife Research Center, Mississippi State University.

\section{Appendix A. Supplementary data}

Supplementary data associated with this article can be found, in the online version, at http://dx.doi.org/10.1016/j.ecolmodel. 2012.03.028.

\section{References}

Bedard, J., 1995. Double-crested cormorant morphometry and field sexing in the St. Lawrence River Estuary. Colonial Waterbirds 18 (Special Publication 1), 86-90.

Blackwell, B.F., Stapanian, M.A., Weseloh, D.V.C., 2002. Dynamics of the double-crested cormorant population on Lake Ontario. Wildlife Society Bulletin 30, 345-353.
Byers, J.A., Castle, S.J., 2005. Areawide models comparing synchronous versus asynchronous treatments for control of dispersing insect pests. Journal of Economic Entomology 98, 1763-1773.

Doligez, B., Cadet, C., Danchin, E., Boulinier, T., 2003. When to use public information for breeding habitat selection? The role of environmental predictability and density dependence. Animal Behaviour 66, 973-988.

Duerr, A.E., 2007. Population Dynamics, Foraging Ecology and Optimal Management of Double-Crested Cormorants on Lake Champlain. University of Vermont, Burlington, USA.

Duerr, A.E., Donovan, T.M., Capen, D.E., 2007. Management-induced reproductive failure and breeding dispersal in double-crested cormorants on Lake Champlain. Journal of Wildlife Management 71, 2565-2574.

Frederiksen, M., Bregnballe, T., 2000. Evidence for density-dependent survival in adult cormorants from a combined analysis of recoveries and resightings. Journal of Animal Ecology 69, 737-752.

Frederiksen, M., Lebreton, J.D., Bregnballe, T., 2001. The interplay between culling and density-dependence in the great cormorant: a modelling approach. Journal of Applied Ecology 38, 617-627.

Guillaumet, A., Dorr, B., Wang, G., Taylor, J.D., Chipman, R.B., Scherr, H., Bowman, J., Abraham, K.F., Doyle, T.J., Cranker, E., 2011. Determinants of local and migratory movements of Great Lakes double-crested cormorants. Behavioral Ecology 22, 1096-1103.

Hansson, B., Bensch, S., Hasselquist, D., Nielsen, B., 2002. Restricted dispersal in a long-distance migrant bird with patchy distribution, the great reed warbler. Oecologia 130, 536-542.

Henaux, V., Bregnballe, T., Lebreton, J.D., 2007. Dispersal and recruitment during population growth in a colonial bird, the great cormorant Phalacrocorax carbo sinensis. Journal of Avian Biology 38, 44-57.

Lande, R., 1977. On comparing coefficients of variation. Systematic Zoology, 214-217.

Lebreton, J.D., Hines, J.E., Pradel, R., Nichols, J.D., Spendelow, J.A., 2003. Estimation by capture-recapture of recruitment and dispersal over several sites. Oikos 101, 253-264.

Lyons, J.E., Runge, M.C., Laskowski, H.P., Kendall, W.L., 2008. Monitoring in the context of structured decision-making and adaptive management. The Journal of Wildlife Management 72, 1683-1692.

Martin, C.A., Alonso, J.C., Alonso, J.A., Palacin, C., Magana, M., Martin, B., 2008. Natal dispersal in great bustards: the effect of sex, local population size and spatial isolation. Journal of Animal Ecology 77, 326-334.

McKee, G.J., 2011. Coordinated pest management decisions in the presence of management externalities: the case of greenhouse whitefly in California-grown strawberries. Agricultural Systems 104, 94-103.

OMNR, 2006. Review of the Status and Management of Double-Crested Cormorants in Ontario. Ontario Ministry of Natural Resource, Fish and Wildlife Branch, Wildlife Section, Peterborough, Ontario.

Parsa, S., Ccanto, R., Rosenheim, J.A., 2011. Resource concentration dilutes a key pest in indigenous potato agriculture. Ecological Applications 21, 539-546.

Pope, L.C., Butlin, R.K., Wilson, G.J., Woodroffe, R., Erven, K., Conyers, C.M., Franklin, T., Delahay, R.J., Cheeseman, C.L., Burke, T., 2007. Genetic evidence that culling increases badger movement: implications for the spread of bovine tuberculosis. Molecular Ecology 16, 4919-4929.

Shonk, K.A., Kevan, S.D., Weseloh, D.V., 2004. The effect of oil spraying on eggs of double-crested cormorants. The Environmentalist 24, 119-124.

Shwiff, S., Kirkpatrick, K., DeVault, T., 2009. The Economic Impact of Double-Crested Cormorants to Central New York. United States Department of Agriculture, Wildlife Services, National Wildlife Research Center, Fort Collins, CO, USA.

Strickland, B.K., Dorr, B.S., Pogmore, F., Nohrenberg, G., Barras, S.C., McConnell, J.E., Gobeille, J., 2011. Effects of management on double-crested cormorant nesting colony fidelity. Journal of Wildlife Management, 1012-1021.

Taylor, J.D., Dorr, B., 2003. Double-crested cormorant impacts to commercial and natural resources. In: Fagerstone, K., Witmer, G. (Eds.), Tenth Wildlife Damage Management Conference Proceedings. Hotsprings, AR, USA, pp. 43-51.

Vreysen, M.J.B., Robinson, A.S., Hendrichs, J., 2007. Area-wide integrated pest management (AW-IPM): principles, practice and prospects. In: Vreysen, M.J.B., Robinson, A.S., Hendrichs, J., Kenmore, P. (Eds.), Area-Wide Control of Insect Pests. Springer, Netherlands, pp. 3-33.

Wires, L.R., Cuthbert, F.J., 2006. Historic populations of the double-crested cormorant (Phalacrocorax auritus): Implications for conservation and management in the 21st century. Waterbirds 29, 9-37.

Zhang, W., van der Werf, W., Swinton, S.M., 2010. Spatially optimal habitat management for enhancing natural control of an invasive agricultural pest: Soybean aphid. Resource and Energy Economics 32, 551-565. 